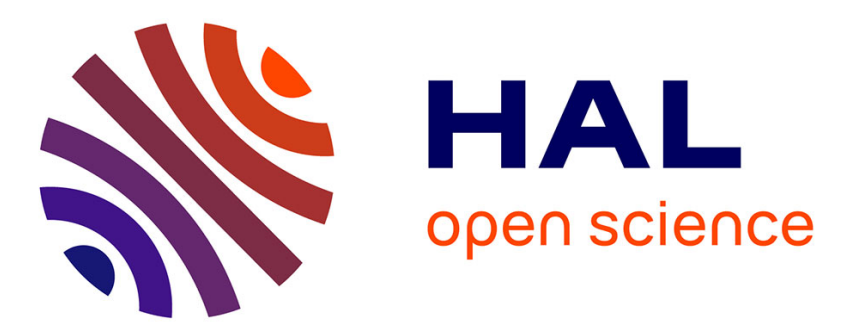

\title{
Anomalous thermal variation of the cobalt anisotropy and longitudinal spin flucuations in $\mathrm{Y} 2 \mathrm{C07}$
}

\author{
Rafik Ballou, L. Lemaire
}

\section{To cite this version:}

Rafik Ballou, L. Lemaire. Anomalous thermal variation of the cobalt anisotropy and longitudinal spin flucuations in Y2C07. Journal de Physique Colloques, 1988, 49 (C8), pp.C8-249-C8-250. 10.1051/jphyscol:19888109 . jpa-00228251

\section{HAL Id: jpa-00228251 https://hal.science/jpa-00228251}

Submitted on 1 Jan 1988

HAL is a multi-disciplinary open access archive for the deposit and dissemination of scientific research documents, whether they are published or not. The documents may come from teaching and research institutions in France or abroad, or from public or private research centers.
L'archive ouverte pluridisciplinaire HAL, est destinée au dépôt et à la diffusion de documents scientifiques de niveau recherche, publiés ou non, émanant des établissements d'enseignement et de recherche français ou étrangers, des laboratoires publics ou privés. 


\title{
ANOMALOUS THERMAL VARIATION OF THE COBALT ANISOTROPY AND LONGITUDINAL SPIN FLUCTUATIONS IN $\mathrm{Y}_{2} \mathrm{Co}_{7}$
}

\author{
R. Ballou and L. Lemaire \\ Laboratoire Louis Néel, C.N.R.S., 166X, 38042 Grenoble cedex, France
}

\begin{abstract}
A single crystal magnetostatic study of the intermetallic compound $\mathrm{Y}_{2} \mathrm{Co}_{7}$ is presented, revealing the existence of extremes in the thermal variation of the anisotropy constants of the compound. These anomalies are understood as resulting from large longitudinal spin fiuctuations which are gradually frozen by an applied field, and associated with metamagnetic instabilities of the cobalt magnetism occurring within a finite range of temperatures.
\end{abstract}

Intermetallic compounds of the Y-Co systems in the composition range from $\mathrm{YCO}_{5}$ up to $\mathrm{YCO}_{2}$ have the following related features: their crystallographic structures are derived from the YCo5 one by the same type of ordered atom substitutions. When this substitution occurs for every three, two or one $\mathrm{YCo}_{5}$ unit cell, the $\mathrm{Y}_{2} \mathrm{Co}_{7}, \mathrm{YCo}_{3}$ and $\mathrm{YCo}_{2}$ phases are generated respectively [1]. Whereas the magnetism of the cobalt in $\mathrm{YCO}_{5}$ keeps its character of well-established ferromagnetism, with magnetization and Curie temperature of the same order as in pure cobalt [2], an exchange enhanced Pauli paramagnetism is observed in $\mathrm{YCo}_{2}$ [2], which undergoes a collective electron metamagnetism in high fields [3]. This collapse is to be associated with a corresponding change in the cobalt atoms coordination in yttrium. From $\mathrm{YCo}_{5}$ to $\mathrm{YCo}_{2}$, this change is gradual and the cobalt atoms are shared out in alternating blocks of two kinds. The first block has the same arrangement as in $\mathrm{YCo}_{5}$ for which the same wellestablished cobalt magnetism should be expected. In the second block, the cobalt atoms have almost the same surroundings as in $\mathrm{YCo}_{2}$ and are then subject to magnetic instabilities. In order to find out whether such instabilities do occur, a single crystal magnetostatic study of the $\mathrm{Y}_{2} \mathrm{Co}_{7}$ compound has been undertaken.

The single crystal had been prepared by a Czochralski method, using high frequency induction technique, in a water cooled copper crucible, under an argon atmosphere. The ingot was checked using $\mathrm{X}$-ray diffraction which revealed it to be single-phased. A crystal of $45 \mathrm{mg}$ with a structure belonging to the space group $\mathrm{R} \overline{\mathrm{m}} \mathrm{m}$ was cut from the ingot by spark erosion. Magnetization measurements were carried out in fields up to $15 \mathrm{~T}$ in the temperature range from $4.2 \mathrm{~K}$ to $420 \mathrm{~K}$ at the Service National des Champs Intenses in Grenoble.

The magnetic isotherms obtained at $4.2 \mathrm{~K}, 240 \mathrm{~K}$ and $400 \mathrm{~K}$ in fields applied parallel and perpendicular to the magnetization axis (trigonal axis of the structure) are reported in figure 1 . In addition to.large magnetocrystalline and magnetization anisotropies, several other peculiarities were observed and are described below.

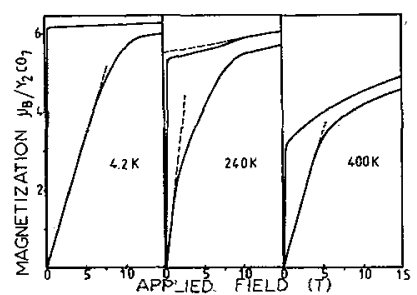

Fig. 1. - Magnetic isotherms measured on the $\mathrm{Y}_{2} \mathrm{Co}_{7}$ single crystal at $4.2 \mathrm{~K}, 240 \mathrm{~K}$ and $400 \mathrm{~K}$ in fields applied respectively parallel and perpendicular to the trigonal axis of the structure.

When the applied field is parallel to the magnetization axis the superimposed differential susceptibility at $240 \mathrm{~K}$ exhibits a maximum around $8 \mathrm{~T}$ suggesting a smooth collective electron metamagnetic transition. On extrapolating the isotherms to zero fields we obtained the thermal variation of the spontaneous magnetization reported in figure 2. It may be seen that the spontaneous magnetization has a positive concave curvature at temperature above $320 \mathrm{~K}$ indicating the end of the process of metamagnetic collapse. The thermal variation of the magnetization measured at $14 \mathrm{~T}$ has also been drawn on the same figure. It exhibits the field stabilizing effect on the cobalt magnetism.

When the applied field is perpendicular to the magnetization axis a quasi-linear variation followed by a bending of the magnetization curves is observed on increasing field. This normally arises from the effects of higher order terms in the expansion of the anisotropy

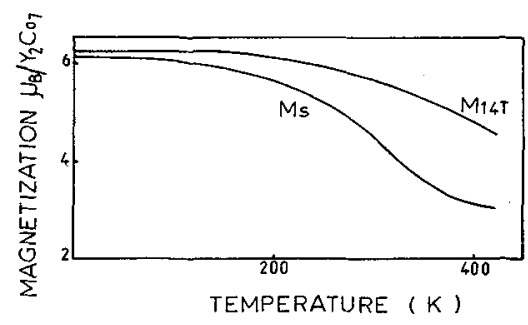

Fig. 2. - Thermal variations of the spontaneous magnetization $M_{\mathrm{s}}$ and the magnetization $M 14 \mathrm{~T}$ measured under an applied field of $14 \mathrm{~T}$ in $\mathrm{Y}_{2} \mathrm{Co}$. 
energy which, according to the cylindrical symmetry of the structure, can be written as:

$$
E_{\mathrm{ani}}=K_{1}(T) \sin ^{2} \theta+K_{2}(T) \sin ^{4} \theta
$$

$\theta$ is the angle between the magnetization vector and the magnetization axis and $K_{1}(T)$ and $K_{2}(T)$ are anisotropy constants respectively responsible for the afore-mentioned linearity and bending.

A visual comparison of the isotherms reported in figure 1 shows that on increasing temperature $K_{1}(T)$ will go to a minimum while $K_{2}(T)$ will have a maximum in the neighbourhood of $240 \mathrm{~K}$ (as indicated by the small range of field over which linearity is observed and the large curvature of the magnetic isotherm at this temperature). These anisotropy constants $K_{1}(T)$ and $K_{2}(T)$ have been quantitatively determined from the measured isotherms using the Sucksmith-Thompson method, taking account of the large observed magnetization anisotropy [4]. Their thermal variations, reported in figure 3 , are strongly correlated, that is where $K_{1}(T)$ shows a decrease, $K_{2}(T)$ increases and conversely.

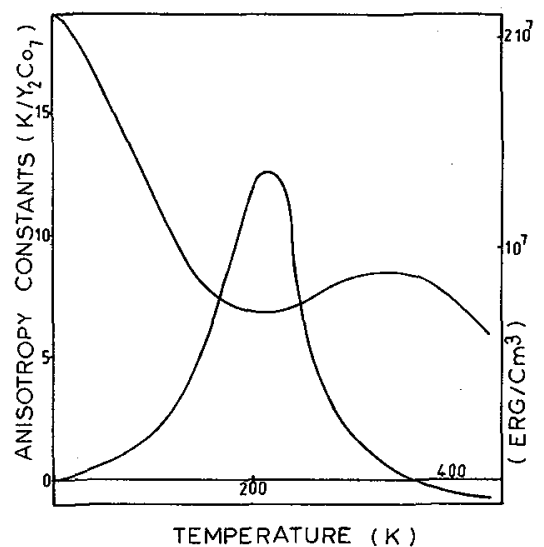

Fig. 3. - Thermal variations of the anisotropy constants in $\mathrm{Y}_{2} \mathrm{Co}$.

Thus, while being in a stable magnetic state at low temperature cobalt magnetism in $\mathrm{Y}_{2} \mathrm{Co}_{7}$ undergoes magnetic instabilities on increasing temperature, leading to a lower magnetization state. This has been confirmed by a polarized neutron diffraction study [5] which also showed that the instabilities concern essentially those cobalt atoms which have almost the same surroundings as in $\mathrm{YCo}_{2}$ whilst those in the $\mathrm{YCo}_{5}$ environment remain magnetically stable. When the in- stabilities occur, the high magnetization state is reinduced by applying a field which gives rise to a maximum in the superimposed differential susceptibility measured along the magnetization axis: a second-order metamagnetic transition takes place through a gradual field freezing of large longitudinal magnetic fluctuations. At higher temperatures the fluctuations are reduced, and the metamagnetic transition ends. The positive curvature in the thermal variation of the spontaneous magnetization observed above $320 \mathrm{~K}$ indicates that the exchange field acting on the cobalt of the $\mathrm{YCo}_{2}$ blocks becomes weak.

Occurrence of large longitudinal spin fluctuations associated to magnetic instabilities in a finite range of temperatures and frozen under applied provides an understanding of the observed thermal variation of the anisotropy constants. These variation, instead of a monotonous decrease expected for a ferromagnet, exhibit extremes which are by no means ascribable to the effect of competing sublattice anisotropy effects. In fact when only thermal transverse magnetic fluctuations are taken into account (the modulus of the magnetization vector being constant [6]) monotonous decreases are predicted. On allowing the modulus to vary as it must in an itinerant system, the extra degree of freedom i.e. longitudinal fluctuations associated to the instabilities will further decrease the anisotropy energy. This explains the minimum of $K_{1}(T)$ at a temperature where the amplitude of the longitudinal fluctuations are the largest. On the other hand, the longitudinal fluctuations are frozen by the application of a field which enables the anisotropy energy to reach again the value that it should have in the absence of any magnetic instabilities. This implies a maximum for $K_{2}(T)$. Consequently the observed anomalies provide characteristic signatures for the instability of the cobalt magnetism in $\mathrm{Y}_{2} \mathrm{Co}_{7}$.

[1] Bertaut, E. F., Lemaire, R. and Schweizer, J., Bull. Soc. Fr. Minér. Crist. 88 (1965) 1445.

[2] Lemaire, R., Cobalt 33 (1966) 201.

[3] Bloch, D., Edwards, D. M., Shimizu, M. and Voiron, J., J. Phys.F. 5 (1975) 1217.

[4] Alameda, J. M., Givord, D., Lemaire, R. and Lu, Q., J. Magn. Magn. Mater. 31 (1983) 191.

[5] Ballou, R. (to be published in Physica B) Proc. Conf. Neutron Scat. (Grenoble) 1988.

[6] Callen, H. H. and Callen, E., J. Phys. Chem. solids 27 (1966) 1271. 